\title{
Modelado y simulación del péndulo de base móvil
}

\section{Modeling and simulation of mobile base pendulum}

\author{
LÓPEZ-ORTEGA, Filemón†**, CÁRDENAS-MACIEL, Martha, SERRANO-ZEPEDA, Rogelio y LARA- \\ CEBALLOS, Miguel Ángel
}

Universidad Tecnológica de Tijuana

ID $1^{\text {er }}$ Autor: Filemón, López-Ortega / ORC ID: 0000-0002-7157-9684, CVU CONACYT ID: 726219

ID $1^{\mathrm{er}}$ Coautor: Martha, Cárdenas-Maciel / ORC ID: 0000-0002-9164-9942

ID $2^{\text {do }}$ Coautor: Rogelio, Serrano-Zepeda / ORC ID: 0000-0002-6209-6402

ID $3^{\text {er }}$ Coautor: Miguel Ángel, Lara-Ceballos / ORC ID: 0000-0002-1803-3009

DOI: $10.35429 /$ JSL.2019.20.6.15.22

Recibido 23 Junio, 2019; Aceptado 30 Septiembre, 2019

\section{Resumen}

El presente artículo describe la simulación y control de un péndulo de base móvil (PBM), que consiste en un mecanismo con dos ruedas y una varilla cilíndrica en vertical, que puede girar libremente sobre su propio eje, entonces el móvil deberá moverse para compensar el desplazamiento angular del péndulo. El objetivo consiste en desarrollar un modelo matemático para simular el comportamiento dinámico del mecanismo y con ello, desarrollar un controlador Proporcional, Integral y Derivativo (PID), óptimo que logre mantener a 90 grados en vertical dicho péndulo en un tiempo $t s \leq 1$ segundo, con un ángulo de entrada de \pm 10 grados. Se utilizó la metodología de Newton-Euler (NE) para determinar las ecuaciones dinámicas de movimiento, mediante el análisis del diagrama de cuerpo libre y el uso de las leyes físicas que permiten definir las fuerzas que actúan sobre el sistema para lograr el estado de equilibrio. Estas simulaciones se realizaron con las herramientas de SolidWorks (SimMechanics Link) y Matlab (Simulink), además se utilizó un sistema de lazo cerrado para analizar la señal de salida $Y(s)$ con respecto a la señal de entrada $\mathrm{U}(\mathrm{s})$. Las contribuciones de este desarrollo consisten en diseñar controladores de alta precisión con el propósito de mejorar los procesos industriales de automatización a partir de la implementación de un sistema de control, en las áreas como robótica, vehículos marinos, aeroespacial, por mencionar algunos ejemplos.

PID, Péndulo, Control

\begin{abstract}
This article describes the simulation and control of a mobile base pendulum (PBM), which consists of a mechanism with two wheels and a vertical cylindrical rod, which can rotate freely on its own axis, then the mobile must move to compensate for the angular displacement of the pendulum. The objective is to develop a mathematical model to simulate the dynamic behavior of the mechanism and thereby develop a Proportional, Integral and Derivative (PID) controller, optimal that manages to maintain this pendulum at a vertical degree in a time ts $\leq$ 1 second, with an entry angle of \pm 10 degrees. The Newton-Euler (NE) methodology was used to determine the dynamic equations of motion, by analyzing the free body diagram and using the physical laws that allow defining the forces acting on the system to achieve the state of equilibrium. These simulations were carried out with the SolidWorks (SimMechanics Link) and Matlab (Simulink) tools, in addition a closed loop system was used to analyze the output signal Y (s) with respect to the input signal U (s). The contributions of this development consist of designing high-precision controllers with the purpose of improving industrial automation processes from the implementation of a control system, in areas such as robotics, marine vehicles, aerospace, to name a few examples.
\end{abstract}

PID, Pendulum, Control

Citación: LÓPEZ-ORTEGA, Filemón, CÁRDENAS-MACIEL, Martha, SERRANO-ZEPEDA, Rogelio y LARA-CEBALLOS, Miguel Ángel. Modelado y simulación del péndulo de base móvil. Revista de Simulación y Laboratorio. 2019, 6-20: 15-22

\footnotetext{
*Correspondencia al Autor (Correo electrónico: filemon.lopez@)uttijuana.edu.mx)

$\dagger$ Investigador contribuyendo como primer Autor
} 


\section{Introducción}

Controlar un sistema significa manipular su respuesta dinámica para cumplir las demandas de una tarea que se va a ejecutar. Por tanto, resulta difícil comprender la respuesta de un modelo dinámico antes de diseñar e implementar un algoritmo de regulación que modifique su comportamiento dinámico. Uno de los métodos más comunes de representación para un esquema de control consiste en dividir la operación por medio de bloques funcionales. Un bloque, como tal, contiene la descripción del comportamiento de cada elemento que actúa en un sistema de control. En particular, el bloque que representa la dinámica del sistema que se va a controlar se denomina comúnmente como planta.

Tomando en cuenta las leyes de la física, se puede deducir el comportamiento de la planta a partir de una ecuación diferencial que define la forma de las variables internas del sistema representadas mediante la ecuación estándar en el dominio de Laplace. El desarrollo del péndulo de base móvil facilitará la comprensión del análisis de la teoría de control y sus aplicaciones en diferentes áreas, como aeroespacial, robótica, biomecánica y transporte, por mencionar algunas.

Este sistema pertenece a la clase de mecanismos subactuados los cuales tienen menor número de actuadores que grados de libertad debido, a su complejidad sigue siendo un campo activo de investigación en el área de control. Este sistema consiste en una barra cilíndrica de longitud $(l)$ con libertad de rotar a $180^{\circ}$ sobre su propio eje, El péndulo está montado mediante un rodillo con cojinete para el libre movimiento sobre un móvil de dos ruedas con desplazamiento lineal en el eje $\mathrm{x}$.

Bajo estas circunstancias el desarrollo de la metodología se enfoca en analizar la dinámica del mecanismo para definir las ecuaciones que describan el movimiento libre del sistema y mediante estas ecuaciones desarrollar un controlador PID que es el elemento de regulación más popular que ha surgido de la teoría de control automático. Es relevante comentar que el controlador PID es habitualmente la primera opción cuando se analiza el diseño de una solución para el sistema de control.
La estructura más convencional de este controlador se puede representar por medio del esquema de bloques en paralelo. Matemáticamente, puede representarse de forma compacta por medio de su expresión en el dominio de Laplace. El control automático ha sido de gran importancia para el desarrollo de la ingeniería y de la ciencia, debido a los avances que se han logrado en la práctica, en la optimización y desempeño de los sistemas dinámicos. A continuación, se presenta el desglose de las secciones del presente artículo. Sección 1: Presenta una breve introducción del análisis del péndulo de base móvil.

Sección 2: Describe la metodología utilizada para modelar matemáticamente la dinámica del péndulo de base móvil a partir de las leyes de Newton-Euler y el desarrollo del controlador (PID) aplicado.

Sección 3: Presenta el desarrollo analítico para determinar la ecuación de la planta, posteriormente se simula en Matlab para conocer la respuesta dinámica del sistema.

Sección 4: Describe el desarrollo del controlador (PID) a partir de la respuesta del modelo y las condiciones sugeridas.

Sección 5: Se presentan los resultados y las conclusiones del presente artículo, así como las mejoras futuras.

\section{Metodología}

Para desarrollar el modelo matemático del péndulo de base móvil, se aplicó la metodología de Newton-Euler (NE), el cual se deriva directamente de la segunda ley de Newton y de los principios de movimiento rotacional planteado por Euler. Fue necesario realizar un diagrama de cuerpo libre de la figura 1, donde se pueden observar cada una de las variables y fuerzas que actúan sobre el mecanismo con movimiento rotacional y traslación. Las condiciones de equilibrio se definen cuando la sumatoria de torques existentes en el sistema es igual a cero y el desplazamiento es nulo. Esta representación se define con una ecuación diferencial que describe matemáticamente el comportamiento dinámico del sistema en el dominio del tiempo. 
Para desarrollar un controlador (PID), es necesario transformar la ecuación diferencial a una función de transferencia, posteriormente a una ecuación estándar representada en el dominio de Laplace, mediante esta ecuación calcular el factor de amortiguamiento ( $\zeta)$, la frecuencia natural amortiguada $(\omega n)$ y el tiempo de establecimiento $(t s)$.

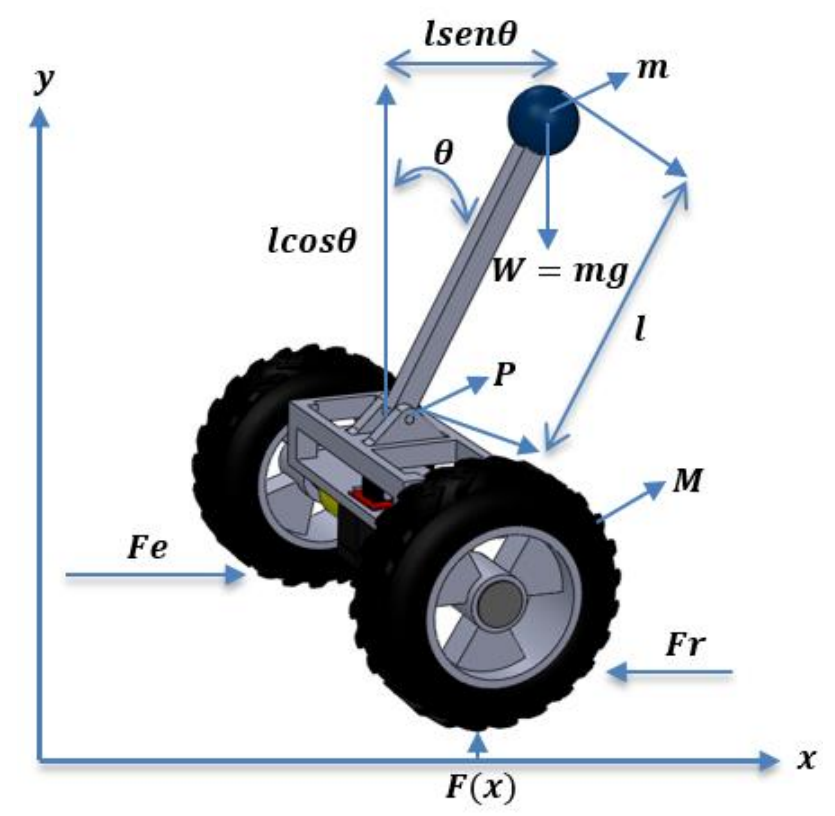

Figura 1 Diagrama de cuerpo libre PBM

En la tabla 1, se describen las variables a considerar de acuerdo a las leyes de Newton a partir del análisis del diagrama de cuerpo libre.

\begin{tabular}{|l|l|}
\hline Símbolos & \multicolumn{1}{c|}{ Descripción } \\
\hline $\boldsymbol{F e}$ & Fuerza de empuje. \\
\hline $\boldsymbol{F r}$ & Fuerza de fricción. \\
\hline $\boldsymbol{l}$ & Longitud de la barra. \\
\hline $\boldsymbol{I}$ & Inercia del péndulo. \\
\hline $\boldsymbol{w}$ & Peso de la esfera. \\
\hline $\mathbf{f}(\mathbf{x})$ & Posición del móvil. \\
\hline $\boldsymbol{\theta}$ & Ángulo del péndulo desde la vertical. \\
\hline $\mathbf{g}$ & Constante gravitacional. \\
\hline $\mathbf{P}$ & Conexión entre los dos sistemas (Origen). \\
\hline $\mathbf{M}$ & Masa de la base móvil. \\
\hline $\mathbf{m}$ & Masa de la esfera. \\
\hline
\end{tabular}

Tabla 2 Descripción de las variables físicas

\section{Modelado matemático del sistema}

La representación matemática del sistema (PBM), se basa en el diagrama de la figura 1, a partir de la definición de un punto de referencia, en este caso, se define como punto de referencia, el centro de gravedad $(\mathrm{Cg})$ del objeto de interés, las ecuaciones (1) y (2), definen el centro de gravedad en las componentes (x) y (y) del péndulo con movimiento rotacional.
En la ecuación (1) el valor de (x) hace referencia al desplazamiento del móvil en cada instante del tiempo o la posición actual.

$x \operatorname{cc}=x+l \operatorname{sen} \theta$

$y c g=l \cos \theta$

Otro término importante en los movimientos rotacionales ya que todo elemento que gira sobre su propio eje posee momento de inercia $(I)$ de acuerdo al Postulado de Euler. Entonces se puede deducir la ecuación (3).

$\sum \tau=I \alpha$

Donde $(\alpha)$ es la aceleración angular del cuerpo y $(\tau)$ es el torque relacionado a la aceleración angular y la fuerza ejercida al eje de giro del sistema. Entonces la aceleración angular ( $\alpha$ ) también se puede representar como $\alpha=$ $\frac{d^{2} \theta}{d t^{2}}$, aplicando la segunda ley de Newton para determinar la ecuación de movimiento rotacional (4), a partir de la ecuación (3). La fuerza de fricción de conexión entre los dos sistemas (Frp), representa la fricción del origen con el eje de giro o el punto de articulación del móvil con el péndulo.

$$
-(F y l \operatorname{sen} \theta+F x l \cos \theta)-F r p=I \alpha
$$



Figura 2 Movimiento del péndulo en Fx y F

El péndulo realiza los movimientos, horizontal $(F x)$ y vertical $(F y)$ como se muestra en la figura 2. Entonces, la expresión $(F x)$ se define en la ecuación 5 y $(F y)$ en la ecuación 6 , la esfera representa el centro de gravedad y (w) define la dirección del vector peso.

$F x=m x c g$

LÓPEZ-ORTEGA, Filemón, CÁRDENAS-MACIEL, Martha, SERRANO-ZEPEDA, Rogelio y LARA-CEBALLOS, Miguel Ángel. Modelado y simulación del péndulo de base móvil. Revista de Simulación y Laboratorio. 2019. 
$F y=m y c g+w$

Para el análisis se prevé que la masa de la esfera se encuentre posicionada en el eje vertical, entonces se deduce que el momento de inercia es igual a cero, por lo que a partir de la ecuación (4), se escribe la ecuación de movimiento rotacional del péndulo, ecuación 7.

$$
(m g l) \operatorname{sen} \theta=m l^{2} \frac{d^{2} \theta}{d t^{2}}+m l \frac{d^{2} x}{d t^{2}} \cos \theta
$$

El objetivo consiste en mantener el péndulo sobre el eje vertical, considerando que los desplazamientos angulares $(\theta)$ son muy pequeños, por lo que $\operatorname{sen} \theta=\theta$ y $\cos \theta=1$ ), entonces es posible linealizar y simplificar la ecuación (7), obteniendo la nueva expresión (8).

$m g \theta=m l \frac{d^{2} \theta}{d t^{2}}+m \frac{d^{2} x}{d t^{2}}$

Para determinar la rapidez de reacción de la base móvil al movimiento rotacional del péndulo, es necesario calcular la fuerza de empuje (Fe) que se aplicará, por lo que, se define en la ecuación 9.

$$
(M+m) \frac{d^{2} x}{d t^{2}}+m l \frac{d^{2} \theta}{d t^{2}}=F e(t)
$$

De las ecuaciones (8) y (9) se aplica el método de eliminación para encontrar las variables $\frac{d^{2} x}{d t^{2}}=\ddot{x} y \frac{d^{2} \theta}{d t^{2}}=\ddot{\theta}$ a continuación, se formulan las siguientes ecuaciones lineales (10).

$$
\begin{aligned}
& m \ddot{x}+m l \ddot{\theta}=m g \theta \\
& (M+m) \ddot{x}+m l \ddot{\theta}=F e(t)
\end{aligned}
$$

Las soluciones del sistema de ecuaciones se presentan en las expresiones (11) y (12).

$$
\begin{aligned}
& \ddot{x}=-\frac{m}{M} g \theta+\frac{1}{M} F e(t) \\
& \ddot{\theta}=\frac{(M+m)}{M l} g \theta-\frac{1}{M l} F e(t)
\end{aligned}
$$

Con la ecuación (12) se determina la función de transferencia del péndulo, la ecuación (13) representa la ecuación diferencial en el dominio del tiempo y las ecuaciones (14), (15) y (16), representan el proceso de la transformada de Laplace para definir la salida $\theta(s)$ en relación a la entrada $F e(s)$, se establecen las condiciones iniciales $\dot{\theta}(0)=0$ y $\theta=0$.

$$
\begin{aligned}
& \frac{d^{2} \theta}{d t^{2}}-\frac{(M+m)}{M l} g \theta=-\frac{1}{M l} F e(t) \\
& s^{2} \theta(s)-g \frac{(M+m)}{M l} \theta(s)=-\frac{1}{M l} F e(s) \\
& \theta(s)\left(s^{2}-g \frac{(M+m)}{M l}\right)=-\frac{1}{M l} F e(s) \\
& \frac{\theta(s)}{F e(s)}=\frac{-\frac{1}{M l}}{s^{2}-g \frac{(M+m)}{M l}}
\end{aligned}
$$

Debido que la velocidad depende de la fuerza de fricción del punto de origen y el eje de giro del sistema, se define la fuerza de fricción del péndulo (Frp) que se muestra en la ecuación (17).

$$
F r p=\beta \frac{d \theta}{d t}
$$

Por lo tanto, el modelo de movimiento rotacional del péndulo en el dominio de la Laplace se representa en la ecuación (18).

$$
\frac{\theta(s)}{F e(s)}=\frac{-\frac{1}{M l}}{s^{2}+\beta s-g \frac{(M+m)}{M l}}
$$

A partir de la ecuación 11, se establecen las condiciones iniciales considerando el estado de equilibrio del móvil, donde $\dot{x}(0)=$ o $y x(0)=0$ y también se hace una aproximación de $f(\theta) \approx f(x)$, para representar la ecuación 11 en la ecuación (19).

$$
\frac{d^{2} x}{d t^{2}}+\frac{m}{M} g x=\frac{1}{M} F e(t)
$$

Se procede a determinar la función de transferencia del móvil que se presentan en las ecuaciones (20) y (21).

$$
\begin{aligned}
& s^{2} X(s)+g \frac{m}{M} X(s)=\frac{1}{M} F e(s) \\
& X(s)\left(s^{2}+g \frac{m}{M}\right)=\frac{1}{M} F e(s)
\end{aligned}
$$

Relacionando la salida y la entrada se obtiene la ecuación (22).

$\frac{X(s)}{F e(s)}=\frac{\frac{1}{M}}{s^{2}+g \frac{m}{M}}$

En la ecuación 22 es conveniente incluir una fuerza de fricción (Fr), la cual es generada por el contacto de las ruedas con el piso, dependiendo del coeficiente de rodamiento, la fuerza de fricción se define en la ecuación 23. 
$F r=\beta \frac{d x}{d t}$

La función de transferencia del movimiento de traslación del móvil, se representa en la ecuación (24).

$$
\frac{X(s)}{F e(s)}=\frac{\frac{1}{M}}{s^{2}+\beta s+g \frac{m}{M}}
$$

\section{Análisis de la respuesta mediante Matlab}

La respuesta de un sistema de control se evalúa por medio de una salida Y(s) que depende de una entrada U(s) y de la dinámica interna instantánea del sistema. Una de las formas de representar un sistema de control es mediante bloques, que consiste en la posibilidad de representar la operación completa de un sistema por medio de la interacción de sus bloques internos. De esta forma, el comportamiento dinámico de una planta que incluye diferentes elementos, como sensores, controladores, retroalimentación, etc., se pueden analizar por medio de bloques representativos y la representación de sus interacciones. Para simplificar la definición de cada elemento funcional, se realiza mediante el operador de la Transformada de Laplace. En la tabla (2) se presentan los valores de las variables a considerar para simular el comportamiento dinámico del péndulo de base móvil mediante las ecuaciones (18) y (24).

\begin{tabular}{|l|l|}
\hline Símbolos & \multicolumn{1}{c|}{ Descripción } \\
\hline $\boldsymbol{M}$ & Masa de la base móvil $=2 \mathrm{~kg}$ \\
\hline $\boldsymbol{m}$ & Masa de la esfera $=0.2 \mathrm{~kg}$ \\
\hline $\boldsymbol{g}$ & Constante gravitacional $=9.81 \mathrm{~m} / \mathrm{s}^{2}$ \\
\hline $\boldsymbol{l}$ & Longitud del péndulo $=0.5 \mathrm{~m}$ \\
\hline $\boldsymbol{\beta}_{\boldsymbol{p}}$ & $\begin{array}{l}\text { Coeficiente de rodamiento para el péndulo } \\
\text { de acero//acero }=0.09 \mu \mathrm{d}\end{array}$ \\
\hline $\boldsymbol{\beta}_{\boldsymbol{M}}$ & $\begin{array}{l}\text { Coeficiente de rodamiento del móvil con el } \\
\text { piso de caucho// cemento }(\mathrm{seco})=0.8 \mu \mathrm{d}\end{array}$ \\
\hline
\end{tabular}

Tabla 3 Variables para simular la respuesta del sistema

De las ecuaciones (18) y (24) al sustituir los valores de acuerdo a las variables de la tabla (2), se obtienen las ecuaciones (25) y (26).

$$
\begin{aligned}
& \frac{\theta(s)}{F e(s)}=\frac{-1}{s^{2}+0.09 s+21.582} \\
& \frac{X(s)}{F e(s)}=\frac{0.5}{s^{2}+0.8 s+0.981}
\end{aligned}
$$

En la gráfica (1) se presenta la respuesta del movimiento rotacional del péndulo a partir de la ecuación (25).

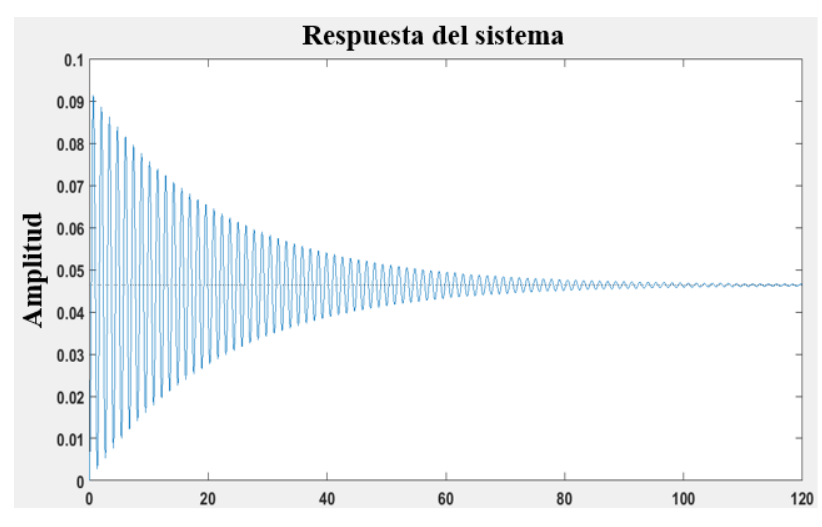

Gráfica 1 Respuesta del sistema péndulo

En la gráfica (2), se presenta el movimiento del móvil a partir de la ecuación (26).

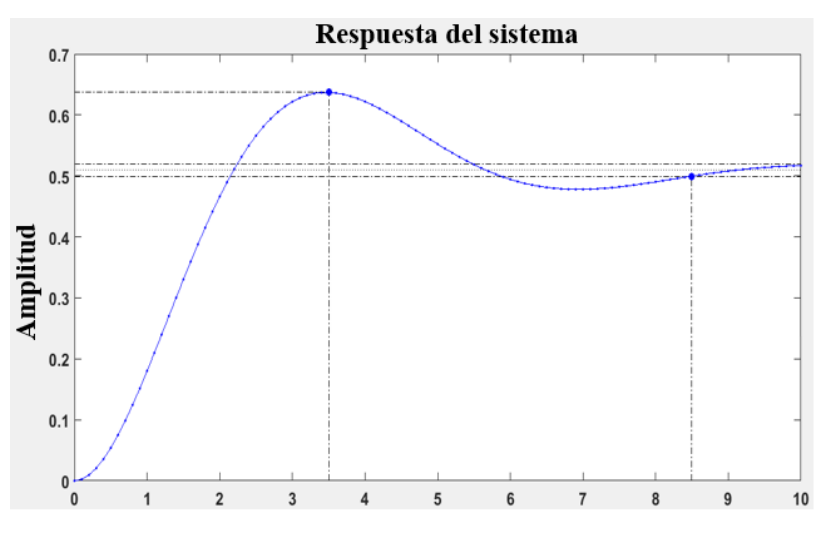

Gráfica 2 Respuesta del sistema base móvil

Mediante la reducción de bloques se determina la función de transferencia general para fusionar el modelo del péndulo la ecuación (25) y el modelo de la base móvil la ecuación (26), la función de transferencia general se expresa como $\mathrm{F}(\mathrm{s})$, ecuación (27).

$F(s)=\frac{0.5}{s^{4}+0.89 s^{3}+22.64 s^{2}+17.35 s+21.17}$

En la gráfica (3) se presenta la respuesta del sistema (PBM) de la ecuación (27).

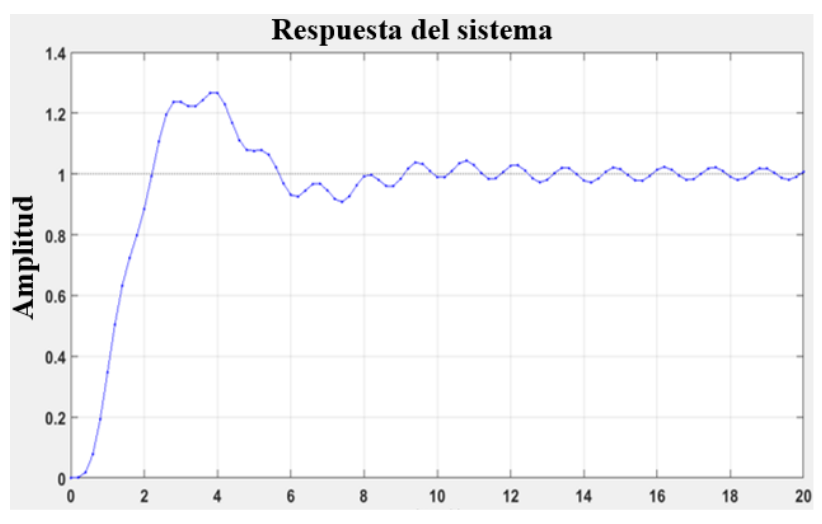

Gráfica 3 Respuesta del sistema PBM

LÓPEZ-ORTEGA, Filemón, CÁRDENAS-MACIEL, Martha, SERRANO-ZEPEDA, Rogelio y LARA-CEBALLOS, Miguel Ángel. Modelado y simulación del péndulo de base móvil. Revista de Simulación y Laboratorio. 2019. 
Mediante Matlab se analiza la respuesta del sistema de la figura (3), se determina que el tiempo de subida $t r=1.39$ segundo, el tiempo de establecimiento $t s=17.6$ segundos, con un sobre impulso $M p=26.6 \%$.

\section{Diseño e implementación del controlador PID}

El controlador Proporcional-Integral-Derivativo (PID), es el elemento de regulación más popular que ha surgido de la teoría de control automático. Es relevante comentar, que el PID es habitualmente la primera opción cuando se analiza el diseño de una solución para el sistema de control. La estructura más convencional de este controlador, se puede representar por medio del esquema de bloques en paralelo como se muestra en la figura 3 , matemáticamente se representa de forma compacta por medio de su expresión en el dominio de Laplace, ecuación (28).



Figura 3 Estructura del PID implementado

$P I D=k p+k i \frac{1}{s}+k d s$

A continuación, se establecen los parámetros para el diseño del controlador de acuerdo a las siguientes condiciones: lograr un tiempo de estabilización $t s \leq 1$ segundo, con un sobre impulso $M P \leq 5 \%$ y un ángulo de entrada $\theta= \pm 10$ grados con respecto a la vertical, de acuerdo a los parámetros de entrada se procede a determinar las constantes del PID mediante el método de sintonización por ubicación de polos y ceros utilizando las funciones de Matlab y la ecuación (28), se determina el modelo del control para el sistema (PBM) en la ecuación 29, donde $k p=0, k i=$ 12.66 y $k d=0$.

$P I D=\frac{12.66}{s}$

En la gráfica 4, se presenta la respuesta del sistema, el color azul representa la salida con la implementación del controlador.
Se determinó que el tiempo de establecimiento $t s=10.8$ segundos, con un sobreimpulso $M P=1.79 \%$, debido a la inestabilidad y complejidad del mecanismo que se desea controlar, el PID, no es lo suficientemente óptimo para lograr uno de los parámetros del objetivo, donde $t s \leq 1 \mathrm{~s}$, se necesita implementar un controlador más robusto que actúe como un filtro para reducir las oscilaciones de la señal de salida y esto permitirá reducir el tiempo de estabilización y posteriormente implementar un PID en caso de ser necesario.

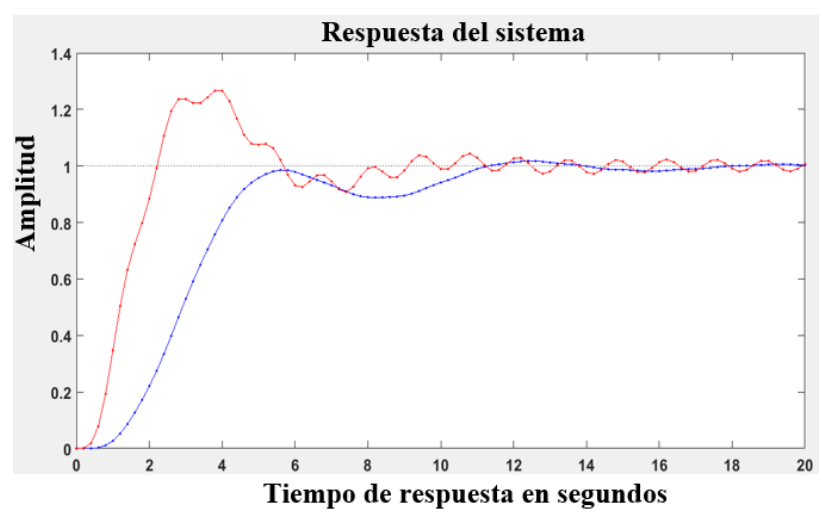

Gráfica 4 Salida del sistema con el PID Implementado

\section{Control Robusto por Modelo Interno}

El método de control robusto por modelo interno (IMC) depende directamente de la estructura del sistema y del modelo matemático de la planta, actúa como un filtro pasa-bajo para reducir las oscilaciones de salida debido a la discrepancia del modelaje y el proceso real, esto permite mejorar el tiempo de respuesta considerablemente, la figura (3) muestra el diagrama de bloques de la estructura de un controlador IMC.

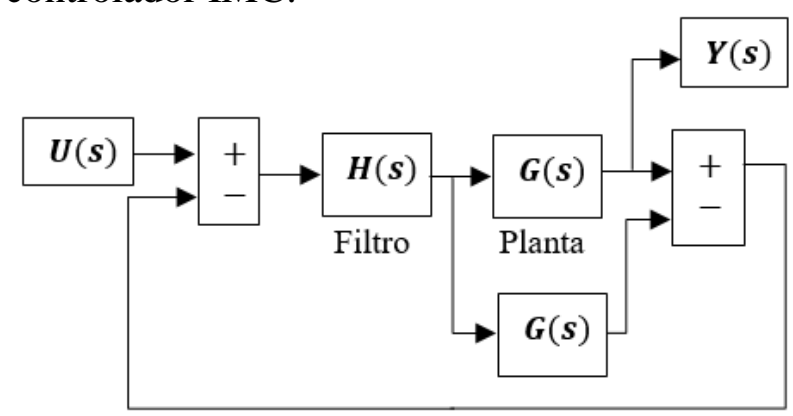

Figura 4 Estructura del controlador IMC

Al implementar el controlador IMC, se puede observar una mejoría considerable en la reducción del tiempo de estabilización de 1.23 segundo y un sobre impulso de $10.8 \%$. gráfica (5), surge la necesidad de incorporar un controlador PID para el modelo actual del IMC.

LÓPEZ-ORTEGA, Filemón, CÁRDENAS-MACIEL, Martha, SERRANO-ZEPEDA, Rogelio y LARA-CEBALLOS, Miguel Ángel. Modelado y simulación del péndulo de base móvil. Revista de Simulación y Laboratorio. 2019. 


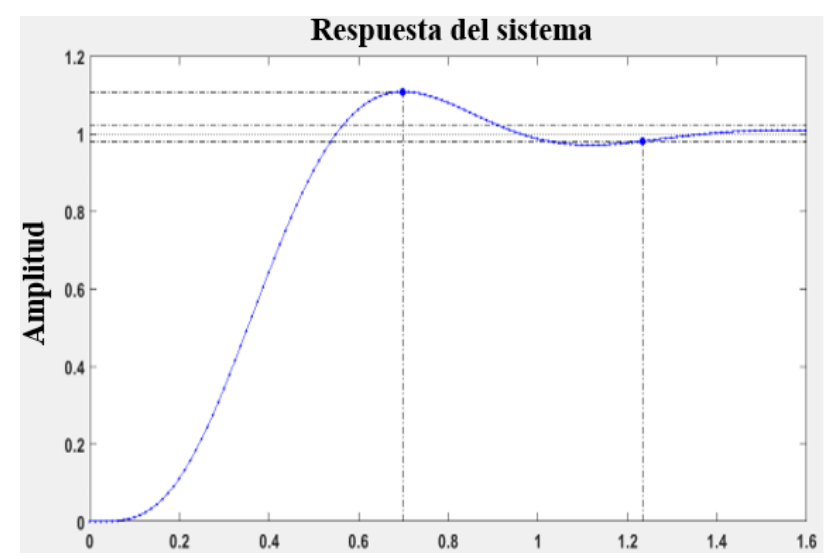

Gráfica 5 Salida del sistema con IMC

A continuación, se determina un controlador PID a partir del modelo IMC, la figura (5) muestra el diagrama de bloques simplificado de la integración del PID al modelo IMC.

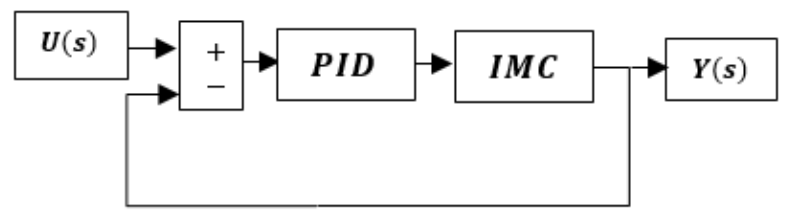

Figura 5 Diagrama de bloques de PID y IMC

\section{Resultados Obtenidos}

Al simular el modelado del sistema PBM con la implementación de los controladores IMC y PID, donde las constantes del PID fueron $k p=$ $0.1209, k i=1.761$ y $k d=0.002076$, se logró el objetivo propuesto con un tiempo de establecimiento $t s=990$ milisegundos y con sobreimpulso $M P=1.79 \%$, en la gráfica (6) el color rojo representa la respuesta del sistema controlada, en la medida que incrementa el grado del filtro aplicado en el control IMC se reduce el tiempo de estabilización (ts).

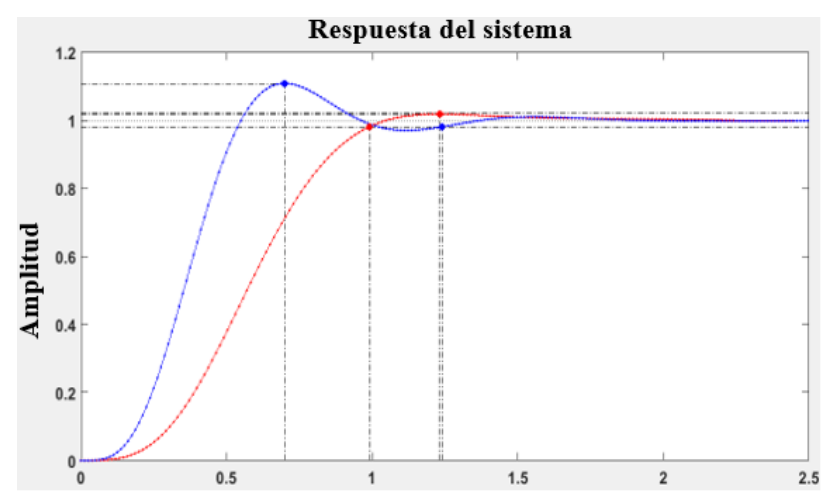

Gráfica 6 Respuesta del sistema con la implementación del y IMC y PID

\section{Conclusiones}

Este artículo presenta el modelo matemático de un péndulo de base móvil, a partir de ecuaciones diferenciales ordinarias no lineales, que se obtuvieron al aplicar las leyes de Newton-Euler y mediante la aplicación de la transformada de Laplace, se obtuvo la función de transferencia que se utilizó para simular y analizar el comportamiento del sistema, con la finalidad de diseñar un controlador que cumpla con el objetivo establecido, para realizar la simulación fue necesario diseñar el mecanismo en SolidWorks y mediante la librería (SimMechanics Link) se vinculó a Matlab y Simulink versión 2017. Con estas herramientas se corroboraron los resultados con los parámetros establecidos en el objetivo, en un principio la idea era diseñar un controlador PID óptimo para el modelo de la planta, pero durante el análisis se determinó que el controlador no cumplía con las expectativas esperadas en cuanto al tiempo de estabilización por tratarse de un sistema muy inestable, entonces se optó primeramente aplicar un controlador robusto al modelo de la plata con el objetivo de reducir las oscilaciones de salida y posteriormente aplicar un PID a la nueva función de transferencia obtenida.

Con estos dos controladores implementados se logró el objetivo propuesto en diseñar, modelar y controlar un péndulo de base móvil, logrando los siguientes resultados $t s=$ $990 m s, M P=1.79 \%$ con un ángulo de entrada $\theta= \pm 10$ grados, la siguiente etapa es poner en práctica el desarrollo analítico, construyendo un prototipo. Con esto se busca mejorar la comprensión en el desarrollo de controladores a partir de modelos matemáticos y que puedan ser usados en sistemas complejos.

\section{Sugerencias}

Es recomendable ajustar los parámetros de un controlador una vez que se lleva a la práctica, ya que estos son diseñados a partir de modelos matemáticos en condiciones ideales y en ocasiones se ven afectados por las variables que no fueron consideradas en el modelado. Otro de los puntos importantes de la dinámica del mecanismo es evitar un alto sobre impulso, ya que este factor tiene la capacidad de desestabilizar completamente el sistema. 


\section{Referencias}

C. Dorf R., H. Bishop R. (2005). Sistemas de control moderno. Madrid: Pearson, Prentice Hall.

Dorsey J. (2005). Sistemas de control continuos y discretos. México: McGraw-Hill.

G. Zill D. (2009). Ecuaciones diferenciales con aplicaciones de modelado. México: Cengage learning.

Hernández Gaviño R. (2010). Introducción a los sistemas de control: Conceptos, aplicaciones y simulación con Matlab. México: Pearson.

J. Aström (2009). Control PID avanzado. Madrid, España: Pearson, Prentice Hall.

J. Craig J. (2006). Robótica. México: Pearson, Prentice Hall.

Ogata K. (1987). Dinámica de Sistemas. Distrito Federal: Prentice Hall

Ogata K. (1998). Ingeniería de Control Moderna. Distrito Federal: Prentice Hall.

Pérez Cisneros M. A., Cuevas Jiménez E.V, \& Zaldívar Navarro D. (2015). Fundamentos de robótica y mecatrónica con Matlab y Simulink. México: Alfaomega.

R. Kurfess T. (2005). Robotics and automation handbook. United States of America: CRC Press.

Reyes Cortes F., (2012). Matlab aplicado a robótica y mecatrónica, México: Alfaomega.

Reyes Cortés F., Cid Monjaraz J., \&Vargas Soto E. (2013). Mecatrónica: Control y automatización. México: Alfaomega. 